\title{
Abstinenzorientierte Behandlungsstrategien im Strafvollzug: Evaluation des Abstinenz-Erprobungs- programms der JVA Vierlande in Hamburg
}

\author{
A. Heinemann \\ K. Bohlen \\ K. Püschel
}

\author{
Drug-free Treatment Strategies in Prison: \\ Evaluation of a Drug-free Programme in Vierlande Prison, Hamburg
}

\section{Zusammenfassung}

Abstinenzorientierte Interventionsmodelle für drogenabhängige Strafgefangene sind sowohl in Deutschland als auch europaweit bislang kaum evaluiert, weder im Hinblick auf ihre Effizienz noch hinsichtlich ihrer Rolle als Vorbereitung für eine Anschlusstherapie. Vorgestellt werden die Ergebnisse einer Langzeitauswertung der Legalbewährung von Teilnehmern eines so genannten Abstinenz-Erprobungsprogramms, das in der Untersuchungshaftund Vollzugsanstalt Vierlande in Hamburg seit 1990 durchgeführt wird. Auf zwei vom übrigen Vollzug außerhalb von Arbeitszeiten getrennten drogenfreien Stationen wird Abstinenz von harten wie weichen Drogen angestrebt und mit täglichen Urinkontrollen überwacht. Unter den Bedingungen von sukzessiven Vollzugslockerungen werden unter fachlicher Betreuung zunehmend lebensweltnahe Bedingungen erprobt, bevor es zur Überleitung in Anschlussmaßnahmen oder Entlassung in Freiheit kommt. In einer Studie wurden 247 männliche Teilnehmer der Jahre 1990 bis 1998 mit einem als Kontrollgruppe definierten Kollektiv von 64 nicht zugelassenen Bewerbern für das Programm hinsichtlich ihrer Hellfeld-Legalbewährung nach Entlassung verglichen (6,2 Jahre durchschnittliche Follow-up-Dauer, Quelle: Bundeszentralregister bei der Generalbundesanwaltschaft). Insgesamt zeigen die Bewerber keine schlechtere Legalbewährung im Hinblick auf Eigentums- und Drogendelikte als die Programmteilnehmer. Teilnehmer, die das Erprobungsprogramm regulär abschlossen, zeigen eine signifikant bessere Legalbewährung als Personen mit vorzeitiger Beendigung. Dabei fallen die Teilnehmer ohne unmittelbar geplante Anschlussmaßnahme gegenüber solchen mit externer Anschlusstherapie durch signifikant kürzere Rezidivlatenzen auf, wobei sich langfristig aber keine höhere Kriminalbelastung ergibt. Frühabbrecher zeigen gegenüber Spätabbrechern eine besonders hohe Kriminalitätsbelastung mit nahezu 100\% Rückfällen im Follow-up. Der Abstinenzerprobungserfolg bestätigt sich als Prädiktor für eine bessere Krimi-

\section{Abstract}

Drug-free treatment in prison for drug dependent inmates has up until now rarely been evaluated in Germany and other European countries. This is particularly true for its important role in a multi stage model with follow-up treatment after release. In this study, the criminal recidivism of prisoners participating in a drug-free programme in the Vierlande prison in Hamburg was evaluated. The programme started in 1990 in two drug-free wards which ensure a separation from the rest of the prisoners for most of the time. The experience of abstinence is supported by a professional therapist in each ward. Prisoners had to obstain from hard and soft drugs and were monitored by daily urine tests. Increasingly realistic life-conditions were being tested in successive loosening of the penitentiary regime before their release. A majority of the successful participants chooses community-based treatment directly after release. Methods: The criminal recidivism of 247 male drug users who participated from 1990 to 1998 was compared to a control group of 64 applicants not admitted to this programme. The mean follow-up period was 6.2 years. The Chief State Prosecutor's register was used for estimating the general and the drug-related delinquency. Results: The control group did not show a worse crime rate in the mean follow-up as well in a standardized 5-years-follow-up period than the participants. Prisoners who finished the programme in a regular way had a significantly lower recidivism rate than drop-outs. Participants who had planned a follow-up treatment after release were significantly longer recidivism-free, but had no advantages on the long-term. A drop out during the first 100 days of treatment predicts a worse outcome with about $100 \%$ criminal recidivism after 5 years. Finishing the programme successfully was a predictor for low criminal recidivism. Looking at the results of recidivism into crime, selection criteria for the rejection of motivated applicants should be considered from a more evidence-based perspective. 
nalprognose. Selektionsmechanismen für behandlungsmotivierte Personen bei der Zulassung zum Programm scheinen allerdings unter dem Evaluationsaspekt der Legalbewährung nicht gerechtfertigt.

Schluiisselwörter:

Opiatabhängigkeit · Abstinenzerprobung · Strafvollzug · Legalbewährung
Key Words:

Opiate Use · Drug-free Programmes · Imprisonment · Criminal Recidivism

\section{Einleitung}

Gefängnisaufenthalte drogenabhängiger Insassen sind eine Herausforderung für das auf Kriminalprävention, aber auch Gesundheitsförderung verpflichtete Strafvollzugssystem. Im Prinzip mag der Gedanke nahe liegen, Abstinenzorientierung sei dem repressiv ausgerichteten Justizvollzugsauftrag systemimmanent. Turnbull [1] beschreibt dies wie folgt: „One of the main reasons why this approach has been adopted within prisons is the perception that prison culture often works against other types of treatment and education". Andererseits widerspricht die reine abstinenzorientierte Ausrichtung jedoch individuell zugeschnittenen rehabilitativen Zielsetzungen sowie dem im Strafvollzugsgesetz festgeschriebenen Grundprinzip der Gleichwertigkeit medizinischer Versorgung innerhalb und außerhalb der Gefängnisse, was im Prinzip nur mit einem breiten Behandlungsangebot unter Einschluss der Substitution vereinbar erscheint. Es gibt darüber hinaus in Deutschland wie in anderen europäischen Staaten nur sehr begrenzte Ressourcen für eine abstinenzorientierte Behandlung im Gefängnis. Dies gilt schon für Entgiftungsbehandlung nach Zugang ins Gefängnis und umso mehr für längerfristige Abstinenzbegleitung in einem geschützten Setting. Sicherlich ist davon auszugehen, dass ein Teil der drogenkonsumierenden Straftäter während eines Gefängnisaufenthalts aufgrund der äußeren Bedingungen - begrenzte Verfügbarkeit der Droge, Konsumvermeidung unter riskanten Bedingungen - unterschiedlich lange Abstinenzperioden durchlebt (Schätzungen belaufen sich auf ca. 50\%). Dabei fehlen jedoch eine individuelle Ausrichtung, eine gezielte Förderung der Abstinenzmotivation und sukzessive Vorbereitung auf die Entlassungssituation zur Vermeidung eines frühen Rückfalls mangels geeigneter, entsprechend personalintensiver Programme.

Es ist eine handlungsleitende Erkenntnis, dass Strafvollzugsaufenthalte per se auch bei unter Haftbedingungen abstinenten Personen keineswegs mit einer geeigneten Vorbereitung auf Abstinenz auch in Freiheit gleichzusetzen sind. Gefängniskulturen können dazu beitragen, die gesundheitliche Situation Drogenabhängiger zu verschlechtern und diese Belastung eher in die Gesellschaft hineinzutragen, als sie von ihr fernzuhalten. Bei der therapeutisch unterstützten Abstinenzorientierung dagegen ist die Zielsetzung, dem dafür motivierten Gefangenen die Möglichkeit zu geben, ein drogenfreies Leben zu erfahren, und zwar zunächst im Schutzraum einer abgeschlossenen Abteilung, dann unter den Bedingungen des Übergangs in die Freiheit, um den Abstinenzentschluss und mittelfristigen Rehabilitationserfolg zu festigen. International existieren auch Konzepte, die mit einer Zwangsrekrutierung von Häftlingen für Abstinenzprogramme arbeiten; in Deutschland ist eine Ausgangsmotivation übliche
Voraussetzung. Die Trennung von den subkulturellen Einflüssen des übrigen Vollzugs ist von konstitutiver Bedeutung ebenso wie Urintestungen als Möglichkeit, Rückfälle zu objektivieren und in einem festen Regelkatalog mit abgestuften Konsequenzen zu belegen. Darüber hinaus gibt es international sehr verschiedene Konzepte der Organisation des Übergangs in die Freiheit für Programmteilnehmer. Zum Teil bestehen diese aus Überleitungsgruppen und auch befristet verpflichtenden kommunalen gruppenorientierten Programmen für die Phase nach Entlassung in die Freiheit, wobei in den USA bislang die umfangreichsten Evaluationsstudien für abgestufte abstinenzorientierte Konzepte für einsitzende Straftäter vorliegen.

80\% der Länder der europäischen Union halten abstinenzorientierte Therapieprogramme vor [1], wobei die Zahl mitgeteilter Therapieplätze offenbar extrem unterschiedlich ist (z. B. Belgien: 16 Plätze in einem flämischen Gefängnis; Spanien: 6456 Teilnehmer in 1999 [2]). Diese sind zumeist mit einem abgestuften Regelwerk aus Rückfall-Sanktionierung und Abstinenzanreizen ausgestattet.

Die Bandbreite des Ressourceneinsatzes für solche Konzepte ist dabei groß. Sie beginnt bei der stringenten Koppelung der Gewährung von Vollzugslockerungen mit dem Nachweis negativer Urinkontrollen [3], also einer mehr disziplinierenden Maßnahme für ein unselektiertes Gefangenenkollektiv. Therapeutische bzw. sekundärpräventive Angebote im engeren Sinne sind zumeist einer von drei Grundkonzeptionen zuzuweisen:

a) „Entgiftungsstationen“ mit einem eher kurzfristigen Behandlungsziel. Ein Beispiel für eine evidenzbasierte Ausrichtung eines Modellprogramms für Entgiftung gibt die Prison Service Order für englische und walisische Gefängnisse (2000) mit Richtlinien u. a. zum Assessment, zur Vernetzung mit therapeutischen Institutionen in Freiheit, zur medikamentösen Stützung und Qualitätskontrolle für Durchführung und Notfallprävention. Basis ist eine Bedarfsdefinition, die von einem Entgiftungsangebot in jedem Gefängnis sowie einer qualitätsssichernden Mitarbeiterschulung ausgeht.

b) „Drogenfreie Stationen“ mit einem unterschiedlichen Anteil an fachlich für die Suchthilfe höherqualifiziertem Personal und somit therapeutischer Begleitung im engeren Sinne sowie verschieden ausgestalteten Behandlungsverträgen mit Verpflichtung der Teilnehmer auf wechselseitige Zusagen. In den Niederlanden gibt es auch verpflichtende drogenfreie Basisprogramme für abhängige Gefangene. 30\% der Teilnehmer schließen dann auf freiwilliger Basis spezialisierte Interventionen an, die dann letztlich von 13\% beendet werden [4]. Als exponiertes Beispiel für ein anspruchsvolles Therapiesetting wird das Kongens-O- 
Pilotprojekt in Vridsleselille/Dänemark genannt [2], das in hohem Maße therapeutisch durchstrukturierte Tagesprogramme und geradezu eine neue Kultur zwischen Insassen und Therapeuten für sich in Anspruch nimmt, allerdings zunächst nur für 15 Teilnehmer konzipiert war. Der Übergang zum Begriff und Selbstverständnis von

c) "therapeutischen Gemeinschaften“ mit Abstinenzorientierung, v.a. im angloamerikanischen Sprachraum, ist also fließend. Eine Teilnahme für 6-12 Monate ist üblich. US-Modelle werden teils in England und Wales übernommen. Manche dieser Programme sind hinsichtlich Reinhaftierungsquoten, Haltequoten oder mittelfristigen Abstinenzerfolgen relativ gut evaluiert. Neuere Projekte kombinieren „in-prison treatment communities“ (ITC) mit nachgeschalteten community-based therapeutic communities und der Betonung der Überlegenheit einer standardisierten Nachsorge (Kyle-New-Vision-Programm in Texas [5, 6]; KEY-CREST-Programm in Delaware [7]; Amity-Programm in Kalifornien [8, 9]). Der Grad der Standardisierung der therapeutischen Intervention (Richtlinienerstellung und Arbeitsmanuale z.B. im Amity-Programm) ist dabei unterschiedlich. Der Akzent liegt auf der abgestuften Sequenz von die jeweilige Rehabilitationsstufe spiegelnden Interventionen (z.B. in Delaware: ITC, FreigängerArbeitsprogramm, Nachsorge nach Entlassung).

In Deutschland gibt es hochschwellig zugängliche sozialtherapeutische Vollzugseinheiten, die sich an speziell sozial rehabilitationsbedürftige wie -fähige Zielgruppen unter den Gefangenen wenden. Hier kommen unter der bereits erbrachten Vorausssetzung gelungener Abstinenzerprobung auch ehemalige Drogenkonsumenten infrage. Trotz der Anteile von Drogenkonsumenten von etwa $20-50 \%$ sind aber nur in einer Minderzahl der Gefängnisse konkret auf diese Zielgruppe zugeschnittene strukturierte Angebote vorhanden, bei denen die Abstinenzhaltung der zu erprobende Rahmen ist, auf dem Gruppenpsychotherapie, Arbeits- und Beschäftigungs- sowie sozial- und sportpädagogische Angebote aufbauen [10].

Die vorliegende Studie konzentriert sich auf ein Programm in der Untersuchungshaft- und Justizvollzugsanstalt Vierlande, das für sich keinen Behandlungsanspruch im engeren Sinne definiert, sondern auf zwei abgeschlosssenen Stationen weitgehend auf Abstinenzerprobung unter fachlicher Begleitung setzt - und im Übrigen einen Teil der Tagesstrukturierung in Form von JVAüblichen Beschäftigungs-/Arbeitseinheiten gewährleistet. Zur Legalbewährung von Teilnehmern in solchen Programmen liegen bislang in Deutschland kaum Daten vor.

\section{Methode}

\section{Das Abstinenzerprobungsprogramm in Vierlande}

Der Senat der Freien und Hansestadt Hamburg beschloss im Jahre 1990 - nach zunehmenden Zahlen drogenabhängiger Insassen die Einrichtung eines modellhaften Abstinenzerprobungsprogramms, das seitdem auf zwei so genannten drogenfreien Stationen mit insgesamt 35 Haftplätzen (reiner Männervollzug) angeboten wird. Vorgesehen ist eine Aufenthaltsdauer von 6-12 Monaten. Bewerber aus Hamburger Vollzugsanstalten werden vom Fachteam aufgesucht. Nach einem persönlichen Gespräch erfolgt die Entscheidung über die Aufnahme im Regelfall im Rahmen einer Fachkonferenz und orientiert sich an „Behandlungsbedarf, -willen und -fähigkeit“. Zielsetzungen sind die Förderung der Selbstkontrolle, wobei im Verlauf des Stationsaufenthalts die Herstellung realistischer Abstinenzbedingungen geplant wird durch Vollzugslockerungen, die Einleitung von Freigangsmaßnahmen mit ambulanter Drogenberatung und die Vorbereitung von Anschlussmaßnahmen gemäß §§35/36 BtmG in Zusammenarbeit mit externen Drogentherapie-Einrichtungen. Jeder Station steht zusätzlich zur vorgesehenen Personalausstattung ein Psychologe, ein Sozialpädogoge und ein Wohngruppenbeamter des Vollzugsdienstes zur Verfügung. Die psychosoziale Betreuung besteht in therapievorbereitenden Einzelgesprächen, die bedarfsabhängig geführt werden. Einmal wöchentlich finden Kleingruppensitzungen statt. Programmatisch stehen hier die Entwicklung drogenfreier Bewältigungs- und Durchsetzungstechniken und soziales Kompetenztraining im Mittelpunkt. Arbeits-, sport- und freizeitpädagogische Maßnahmen ergänzen das Angebot. Ein durchstrukturiertes therapeutisches Setting im engeren Sinne mit einer festgelegten Interventionsphilosophie bzw. individuellen Therapieplänen besteht ausdrücklich nicht. Im Mittelpunkt des Angebots steht das strukturierende Moment, wobei das Urinkontrollprogramm einen zentralen Stellenwert einnimmt. Es erfolgen konsequent tägliche Urinkontrollen. Nachgewiesene Clean-Phasen von mehr als drei Monaten Dauer führen bei Vorliegen der vollzuglichen Voraussetzungen zu Vollzugslockerungen, Rückfälle dagegen nach einem transparenten Regelsystem zu deren Rücknahme. Im Prinzip gelten drei positive Urinkontrollen innerhalb eines Behandlungsmonats als Ausschlusskriterium. Grundsätzlich wird dabei zwischen weichen und harten Drogen nicht unterschieden. Das Programm versteht sich als Therapievorbereitung. Bei Personen, bei denen eine therapeutische Weiterentwicklung nicht erforderlich erscheint oder nicht angestrebt wird, stehen Rückfallprävention und Entlassungsvorbereitung im Mittelpunkt [11].

\section{Untersuchungskollektiv}

In die Studie wurden 408 männliche Programmteilnehmer mit Eintritt bis Ende 2000 aufgenommen, von denen eine Behandlungsdokumentation in Form von Urinkontrollverlaufsbögen archiviert war. 55 Personen hatten zwei, 26 drei Therapieereignisse im Untersuchungszeitraum absolviert. Bei der Auswertung der Legalbewährung wurde ggf. das jeweils letzte Therapieereignis als Beginn des Follow-ups gewertet. Das Kollektiv für die Analyse der Legalbewährung wurde auf 291 Personen mit letztem Therapieereignis bis 1998 beschränkt. Als Kontrollgruppe diente ein Kollektiv von 72 bis 1995 abgelehnten Bewerbern für das Programm, bei denen von einer Teilnahmemotivation zumindest insoweit auszugehen war, als sie über die Bewerbung hinaus ein Auswahlgespräch gehabt hatten und erst in der Fachkonferenz eine Negativentscheidung gefallen war. Über die Teilnehmer wurden Alter, Teilnahmedauer, Art und Gründe des Abstinenzerprobungsendes erhoben, außerdem Anschlusstherapiemaßnahmen, sämtliche Haftzeiten seit 1987 und die Monatsprävalenz positiver Urinkontrollen für weiche und harte Drogen. Als Datenquellen dienten die Teilnehmerdokumentationen sowie das Hamburger Strafvollzugs-Zentralregister. 
Vom Bundeszentralregister beim Generalbundesanwalt wurden Anfang 2002 pseudonymisierte, vollständige Strafregisterauszüge eingeholt. Die Studie erfolgte auf der Grundlage eines genehmigten Datenschutzkonzepts im Einverständnis mit dem Strafvollzugsamt der Justizbehörde der Freien und Hansestadt Hamburg.

Von 311 der angefragten 363 Personen wurden Registerauszüge erhalten (Ausschlussgründe: Auskunftssperre, Tod, Abschiebehäftlinge oder keine eindeutige Zuordnung möglich auf Basis von Namen, Geburtsdatum, Geburtsort). Es lagen auswertungsfähige Datensätze von 134 Teilnehmern mit erfolgreichem Abschluss der Abstinenzerprobung, 113 Abbrechern sowie 64 abgelehnten Bewerbern (Kontrollgruppe) vor. Die Auswertung bezog aufgrund der Meldeverzögerung nur Eintragungen bis 2000 ein und umfasste eine durchschnittliche Follow-up-Dauer von 6,2 Jahren (haftzeitbereinigt 4,9 Jahre, StAbw 2,5 Jahre, Range 0,5-10 Jahre $\left.^{1}\right)$.

Die Analyse konzentrierte sich auf folgende Strategien:

d) Berechnung von Rückfallraten hinsichtlich der Gesamtkriminalität (sämtliche Delikte des StGB sowie BtmG) einerseits, spezifischer Drogenkriminalität andererseits. Als Konstrukt für „Drogenkriminalität“ im engeren Sinne wurden in dieser Studie neben BtmG-Delikten sämtliche Eigentumsdelikte nach StGB gewertet, um direkte wie indirekte Beschaffungskriminalität, wenn auch mit begrenzter Spezifität, mitzuerfassen.

e) Berechnung von Bewährungsindices als Deliktfrequenz pro Jahr in Freiheit für den Zeitraum vor und nach jenem Haftaufenthalt, in den die Programmteilnahme oder (im Fall der Kontrollgruppe) Syntax die Bewerbung integriert war. Berechnung des Verhältnisses zwischen Bewährungsindex vor und nach Intervention/Bewerbung (Ratio).

f) Ermittlung des Trends: Absenkung/Stabilität der Straffälligkeitsfrequenz während verbrachter Zeit in Freiheit im Vergleich zum Zeitraum vor Intervention/Bewerbung: Trend positiv; bei Steigerung: Trend negativ ${ }^{2}$.

g) Berechnung von einheitlichen 5-Jahres-Rückfallraten für eine Teilstichprobe von $\mathrm{n}=210$ Personen, bei denen ein individueller 5-Jahres-Längsschnitt möglich war (Haftende nach Teilnahme/Bewerbung nicht später als 31.12.95, individuelles Follow-up 5 Jahre, keine Haftzeitkorrektur des Beobachtungszeitraums).

Die statistische Auswertung erfolgte mit SPSS 10.0. Die Verteilung dichotomer/kategorialer Variablen wurde mit nicht parametrischen (Chi-Quadrat), bei kontinuierlichen Parametern mit ANOVA-Testverfahren univariat gegen Kontrollgruppe getestet

\footnotetext{
1 Mehrere Personen mit durchgehendem Haftaufenthalt im Follow-up ohne Entlassung in Freiheit waren wiederholt - offenbar im Rahmen von Ausgängen/Hafturlauben - straffällig geworden, so dass für quantitative Auswertungen der Mindestwert der Bewährungszeit mit 0,5 Jahre gesetzt wurde.
}

2 Die Auswertungen, die auf einen Vorher/Nachher-Vergleich abstellen sind nicht kontrollierbar hinsichtlich der Längsschnitt-Verzerrung durch evtl. Löschungen von Registereinträgen nach 5-jähriger (u.a. Freiheitsstrafen bis drei Monate), 10-jähriger oder 15-jähriger Löschungsfrist (siehe BZRG §46).
(Signifikanzniveau p = 0,05). Mit logistischer Regression wurden Teilnahmedauer, Kriminalitäts-Vorbelastung sowie Frequenz positiver Urinkontrollen (Heroin, Kokain, Cannabis)während des Programms auf ihre prädiktive Qualität für eine positive Legalbewährung getestet.

\section{Ergebnisse}

Programmteilnehmer und Kontrollgruppe zeigen von der Altersstruktur und der quantitativen Straftatvorbelastung her keine er-

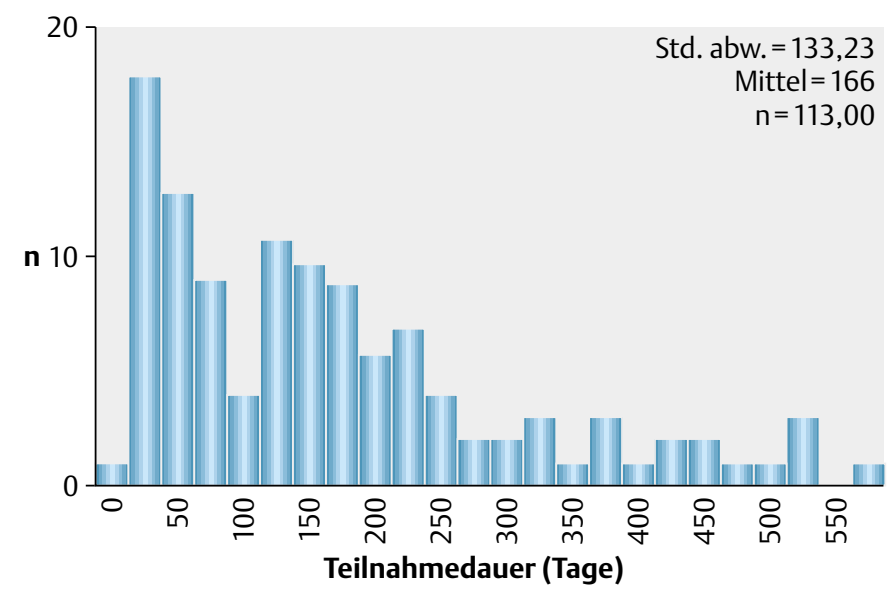

kennbaren Strukturunterschiede (Tab.1). Die Teilnahmedauer liegt bei Programmabbrechern im Mittel nur 63 Tage unter jener der erfolgreichen Absolventen (229 Tage). Die Verteilung der Teilnahmedauern unter den Abbrechern stellt sich zweigipflig dar mit einem ersten Schwerpunkt im Bereich der ersten 100 Tage (Abb.1). Früh- und Spätabbrecher (>100 Tage) werden im Folgenden getrennt betrachtet.

Die Follow-up-Analyse des gesamten Datenmaterials mit individuell unterschiedlichen Beobachtungsdauern nach Programmteilnahme zeigt, dass hinsichtlich der Legalbewährung nach der Therapie nur ein geringer Teil des Untersuchungskollektivs rezidivfrei blieb, wobei sich die Werte zwischen Programmteilnehmern und Kontrollgruppe nicht signifikant unterscheiden (Tab. 2), erstere mit insgesamt ca. 16\% Rückfallfreien in der Drogenkriminalität sogar unterhalb der Kontrollgruppe (ca. 20\%) abschneiden. Nach Therapieabbruch, insbesondere in den ersten 100 Tagen, blieb allerdings nur eine einzige Person rezidivfrei, die Teilnehmer mit regulärer Beendigung schneiden signifikant besser ab (23\% rezidivfrei). Auch eine standardisierte 5-JahresFollow-up-Analyse mit einer Substichprobe von Personen, die nicht später als 31.12 .95 entlassen wurden, zeigt ähnliche, von der Tendenz für die Kontrollgruppe noch leicht verbesserte Werte.

Ein differenzierteres Bild ergibt sich hinsichtlich des Bewährungstrends im Vergleich zur Straffälligkeitsfrequenz pro Jahr in Freiheit vor und nach der Intervention bzw. Bewerbung, da so mit der individuellen Legal-Vorbelastung verglichen wird (qualitative Betrachtung, Tab.3). Hier gibt es zwischen den Erfolgreichen und der Kontrollgruppe wiederum keinen signifikanten Unterschied, allenfalls einen statistischen Trend (bei potenziell spezifisch drogenbezogener Kriminalität $p=0,17$, bei der Gesamtdeliktfrequenz nach StGB p =0,11). Teilnehmer mit regu- 
Tab.1 Strukturdaten im Subgruppenvergleich

\begin{tabular}{|c|c|c|c|c|c|}
\hline Fälle/Kontrollen & Subgruppe & & $\begin{array}{l}\text { Alter bei } \\
\text { Therapiebeginn }\end{array}$ & $\begin{array}{l}\text { Verurteilungen vor } \\
\text { Therapie/Bewerb. (n) }\end{array}$ & $\begin{array}{l}\text { Therapiedauer } \\
\text { (Tage) }\end{array}$ \\
\hline \multirow[t]{15}{*}{$\begin{array}{l}\text { Programm- } \\
\text { teilnehmer }\end{array}$} & $\begin{array}{l}\text { Programmteilnehmer } \\
\text { mit regulärem Abschluss }(n=134)\end{array}$ & Mittelwert & 28,9 & 6,8 & 229,3 \\
\hline & & Standardabw. & 4,2 & 3,7 & 124,5 \\
\hline & & Median & 28,2 & 6 & 212 \\
\hline & & Minimum & 21,6 & 1 & 31 \\
\hline & & Maximum & 44,9 & 18 & 679 \\
\hline & Programm-Abbrecher $(n=113)$ & Mittelwert & 29,6 & 8,9 & 166,2 \\
\hline & & Standardabw. & 4,5 & 3,8 & 133,2 \\
\hline & & Median & 29,1 & 9 & 143 \\
\hline & & Minimum & 21,8 & 1 & 7 \\
\hline & & Maximum & 43,3 & 19 & 575 \\
\hline & Teilnehmer insgesamt & Mittelwert & 29,2 & 7,8 & 200,5 \\
\hline & & Standardabw. & 4,3 & 3,9 & 132,1 \\
\hline & & Median & 28,6 & 8 & 175 \\
\hline & & Minimum & 21,6 & 1 & 7 \\
\hline & & Maximum & 44,9 & 19 & 679 \\
\hline \multirow[t]{5}{*}{ Kontrollgruppe } & abgelehnte Bewerber $(n=64)$ & Mittelwert & 29,3 & 8,0 & \\
\hline & & Standardabw. & 5,3 & 3,8 & \\
\hline & & Median & 28,4 & 8 & \\
\hline & & Minimum & 21,4 & 1 & \\
\hline & & Maximum & 47,1 & 19 & \\
\hline \multirow[t]{5}{*}{ insgesamt } & insgesamt $(n=311)$ & Mittelwert & 29,2 & 7,8 & 200,5 \\
\hline & & Standardabw. & 4,6 & 3,9 & 132,1 \\
\hline & & Median & 28,6 & 8 & 175 \\
\hline & & Minimum & 21,4 & 1 & 7 \\
\hline & & Maximum & 47,1 & 19 & 679 \\
\hline
\end{tabular}

Tab.2 Anteile rezidivfreier Personen bei drogenbezogener/Gesamtkriminalität

(a) Gesamt-Follow-up, im Mittel 6,2 Jahre, $n=311$

(b) einheitliches 5-Jahres-Follow-up einer Substichprobe, $n=210$

\begin{tabular}{|c|c|c|c|c|}
\hline & \multicolumn{2}{|c|}{ drogenbezogene Kriminalität } & \multicolumn{2}{|l|}{ Gesamtkriminalität } \\
\hline & Gesamt-Follow-up & 5 Jahre & Gesamt-Follow-up & 5 Jahre \\
\hline Programmteilnehmer insgesamt & $15,8 \%$ & $15,3 \%$ & $12,1 \%$ & $13,4 \%$ \\
\hline Programmende regulär, ext. Therapieanschluss & $23,7 \%$ & $27,0 \%$ & $18,3 \%$ & $22,2 \%$ \\
\hline Programmende regulär, ohne ext. Therapieanschluss & $22,0 \%$ & $15,8 \%$ & $19,5 \%$ & $15,8 \%$ \\
\hline Abbruch erste 100 Tage & $2,3 \%$ & $3,2 \%$ & $2,3 \%$ & $3,2 \%$ \\
\hline Abbruch nach > 100 Tagen & $10,0 \%$ & $6,8 \%$ & $5,7 \%$ & $6,8 \%$ \\
\hline abgelehnte Bewerber & $20,3 \%$ & $24,5 \%$ & $14,1 \%$ & $18,9 \%$ \\
\hline
\end{tabular}

lärem Abschluss, aber ohne Anschlusstherapie zeigen keine schlechtere Trendentwicklung der Legalbewährung als mit Anschluss, gerade in Bezug auf die Drogenkriminalität.

Teilnehmer mit regulärem Programmende weisen gegenüber Abbrechern signifikant bessere Bewährungstrends hinsichtlich der Verurteilungsfrequenz nach StGB/BtmG $(\mathrm{p}=0,07)$, der Deliktfrequenz nach StGB (alle Strafttatbestände, $p=0,025$ ), der Deliktfrequenz nach BtmG $(p=0,005)$ und auch hinsichtlich der gesamten drogenbezogenen Kriminalität $(p=0,047)$ auf.
Bei den Abbrechern verschlechtert sich die drogenbezogene Deliktfrequenz immerhin in der Hälfte der Fälle nicht, die Gesamtkriminalität zeigt allerdings überwiegend eine Verschlechterung. Vorher/Nachher-Betrachtungen sind zwar nur eingeschränkt zulässig: Die Voraussetzungen für Löschungen länger zurückliegender Delikte im BZR dürften aber angesichts der hohen Rezidivrate nur selten vorgelegen haben.

Eine quantitative Betrachtung erbringt ähnliche Ergebnisse: Zwischen den Mittelwerten der einzelnen Subgruppen unter den Programmteilnehmern sowie dem Mittelwert der Kontrollgruppe zeigen sich keine signifikanten Unterschiede. Mit jährlich 1,38 Delikten innerhalb des hier gewählten Konstrukts „Drogen- 
Tab.3 Personen mit positivem Entwicklungstrend der Legalbewährung (Verlaufsbetrachtung der Deliktfrequenz für Gesamt- bzw. Drogenkriminalität pro Jahr in Freiheit vor und nach Programmteilnahme bzw. Bewerbung)

\begin{tabular}{|c|c|c|c|c|c|c|}
\hline & \multicolumn{2}{|c|}{$\begin{array}{l}\text { Gesamtkriminalität: } \\
\text { Positivtrend } \\
\text { Verurteilungen/Jahr }\end{array}$} & \multicolumn{2}{|c|}{$\begin{array}{l}\text { Gesamtkriminalität: } \\
\text { Positivtrend Delikte/Jahr, } \\
\text { haftzeitkorrigiert }\end{array}$} & \multicolumn{2}{|c|}{$\begin{array}{l}\text { Drogenkriminalität: } \\
\text { Positivtrend Delikte/Jahr, } \\
\text { haftzeitkorrigiert }\end{array}$} \\
\hline & Anzahl & $\%$ & Anzahl & $\%$ & Anzahl & $\%$ \\
\hline $\begin{array}{l}\text { Programmteilnehmer } \\
\text { insgesamt }\end{array}$ & 179 & $72,5 \%$ & 127 & $51,4 \%$ & 142 & $57,5 \%$ \\
\hline $\begin{array}{l}\text { Programmende regulär, } \\
\text { ext. Therapieanschluss }\end{array}$ & 69 & $74,2 \%$ & 56 & $60,2 \%$ & 57 & $61,3 \%$ \\
\hline $\begin{array}{l}\text { Abbruch } \\
\text { erste } 100 \text { Tage }\end{array}$ & 28 & $65,1 \%$ & 19 & $44,2 \%$ & 22 & $51,2 \%$ \\
\hline $\begin{array}{l}\text { Abbruch } \\
\text { nach > } 100 \text { Tagen }\end{array}$ & 54 & $77,1 \%$ & 29 & $41,4 \%$ & 36 & $51,4 \%$ \\
\hline $\begin{array}{l}\text { abgelehnte } \\
\text { Bewerber }\end{array}$ & 48 & $75,0 \%$ & 32 & $50,0 \%$ & 35 & $54,7 \%$ \\
\hline
\end{tabular}

Tab. 4 Quantitativer Follow-up-Vergleich: Drogenkriminalität in Delikten/Jahr in Freiheit (Signifikanztestung gegen Kontrollgruppe)

\begin{tabular}{|c|c|c|c|c|c|}
\hline & & $\begin{array}{l}\text { Follow-up: } \\
\text { Drogenkriminalität: } \\
\text { Delikte/Jahr }\end{array}$ & $p^{*}$ & $\begin{array}{l}\text { Drogenkriminalität: } \\
\text { Ratio nachher/vorher }\end{array}$ & $p$ \\
\hline \multirow[t]{3}{*}{ Programmteilnehmer insgesamt } & Mittelwert & 1,38 & 0,45 & 1,11 & 0,89 \\
\hline & Standardabw. & 2,08 & & 1,58 & \\
\hline & Median & 0,7 & & 0,45 & \\
\hline \multirow[t]{3}{*}{ Programmende regulär, ext. Therapieanschluss } & Mittelwert & 1,45 & 0,92 & 1,18 & 0,7 \\
\hline & Standardabw. & 2,26 & & 1,71 & \\
\hline & Median & 0,74 & & 0,48 & \\
\hline \multirow[t]{3}{*}{ Programmende regulär, ohne ext. Therapieanschluss } & Mittelwert & $1,22^{*}$ & 0,45 & 0,95 & 0,25 \\
\hline & Standardabw. & 1,62 & & 1,28 & \\
\hline & Median & 0,64 & & 0,34 & \\
\hline \multirow[t]{3}{*}{ Abbruch erste 100 Tage } & Mittelwert & 2,21 & 0,09 & 1,76 & 0,22 \\
\hline & Standardabw. & 2,64 & & 1,88 & \\
\hline & Median & 1,41 & & 0,97 & \\
\hline \multirow[t]{3}{*}{ Abbruch nach > 100 Tagen } & Mittelwert & 1,88 & 0,25 & 1,46 & 0,51 \\
\hline & Standardabw. & 2,11 & & 1,78 & \\
\hline & Median & 1,06 & & 0,98 & \\
\hline \multirow[t]{3}{*}{ Kontrollgruppe: abgelehnte Bewerber } & Mittelwert & 1,48 & & 1,28 & \\
\hline & Standardabw. & 1,85 & & 1,46 & \\
\hline & Median & 0,86 & & 0,78 & \\
\hline \multirow[t]{3}{*}{ insgesamt } & Mittelwert & 1,63 & & 1,3 & \\
\hline & Standardabw. & 2,14 & & 1,65 & \\
\hline & Median & 0,9 & & 0,73 & \\
\hline
\end{tabular}

${ }^{*} \mathrm{p}=0,047$ gegenüber Frühabbrechern in den ersten 100 Tagen

kriminalität" liegen die Programmteilnehmer im AnschlussFollow-up insgesamt nur unwesentlich unter der Straffälligkeit der Kontrollgruppe. Gleiches gilt für das Verhältnis der Deliktfrequenz im Zeitraum nach Programmteilnahme zu Deliktfrequenz vor Programmteilnahme (retrograde Betrachtung reicht dabei nur bis 1987 zurück, da darüber hinaus keine Haftzeitbereinigung möglich). Ob ein regulärer Programmabschluss mit oder ohne geplanten Therapieanschluss, z. B. nach $\S \S 35$ ff BtMG, stattfand, hat keine signifikante Auswirkung auf die Legalbewährung, Personen ohne stehen mit 1,22 Delikten/Jahr hier allerdings tendenziell etwas besser da als solche mit Anschluss- therapie (1,45 Delikte/Jahr). Die Subgruppe ohne geplante Anschlussmaßnahme zeigt eine signifikant bessere Bewährung als die der Frühabbrecher $(p=0,045)$.

Ein weiterer Parameter der Legalbewährung ist die Zeit von der Haftentlassung bis zum ersten deliktischen Rückfall (Tab.5). Gegenüber der Kontrollgruppe zeigten Programmteilnehmer mit externem Therapieanschluss eine knapp an der Signifikanzschwelle liegende Verlängerung der rezidivfreien Zeit (im Mittel 517 Tage zu 343 Tagen bzw. im Median 338 zu 129 Tagen). Nur auf Drogenkriminalität bezogen, war die Rückfalllatenz weniger 
deutlich von der Kontrollgruppe abgesetzt. Weiter zeigten sich signifikant schnellere Rückfälle (Gesamt- wie spez. Drogenkriminalität) bei Personen ohne geplante Anschlussbehandlung.

Welche Indikatoren sprechen bei Personen mit regulärem Programmabschluss für Rezidivfreiheit in der Legalbewährung? Univariat scheidet das Lebensalter bei Therapiebeginn aus. In der binär logistischen Regressionsanalyse zeigen eine längere Therapiedauer und eine geringere Vorstrafenbelastung einen schwach positiven Einfluss. Der fehlende Nachweis harter Drogen während des Programms erweist sich als stärkster Prädiktor einer positiven Legalprognose.

Der Median der rückfallfreien Zeit liegt bei unter 25-Jährigen bei 353 Tagen, bei über 30-Jährigen bei 221 Tagen. Auch Mittelwert und Median der drogenbezogenen Delikte pro Jahr in Freiheit im Follow-up steigen mit zunehmendem Alter an. Eine längere Therapiedauer hat keinen einheitlichen Effekt. Oberhalb des 4. Quartils (288 Tage) ist sie mit der besten Quote für Rezidivfreiheit verbunden. Wenn Langzeitteilnehmer allerdings rückfällig werden, dann besonders früh (Abb. 2), sicher auch deshalb, weil bei langfristiger Teilnahme in 50\% der Fälle auf eine Anschlusstherapie verzichtet wird (Teilnehmer mit regulärem Abschluss bis 100 Tage: 10\%).

\section{Diskussion}

Die Ergebnisse unterstützen, dass es sich bei der betreuten Abstinenzerprobung in der JVA Vierlande aus Sicht der Sekundärprävention um eine geeignete Vorbereitung entweder einer Anschlusstherapiemaßnahme oder aber um eine Vorbereitung auf die besonders rückfallgefährdete Entlassungssituation handeln kann. Die retrospektive Längsschnittanalyse kann aber nicht nachweisen, dass die Maßnahme als solche bereits einen eigenen langfristig stabilisierenden Effekt auf die Legalbewährung hat. Dabei ist zu beachten, dass die Kontrollgruppe im Sinne einer konservativen Betrachtung ausgewählt wurde, da hier von einer klar formulierten Abstinenzmotivation auszugehen war. Wenn man annimmt, dass die untersuchte Selektion von Delikten direkter und indirekter Drogenkriminalität sehr spezifisch eine fortgesetzte Drogenkarriere und nur selten eine vom Beschaffungsdruck unabhängige Habitualisierung straffälliger Verhaltensweisen spiegelt, ist die Legalbewährung ein Parameter auch für den Abstinenzerfolg. So erscheinen die Rückfallraten in den Drogenkonsum im langjährigen Verlauf relativ hoch. Es zeigt sich aber, dass bereits die Frage, ob eine Abstinenzerprobung unter Haftbedingungen regulär abgeschlosssen wird oder nicht, eine prädiktive Funktion für die Legalbewährung besitzt, insbesondere beim frühen vorzeitigen Abbruch. Bei regulärer Beendigung des Programms ist im Langzeitverlauf keine geringere Straffälligkeit für Personen mit geplanten Anschlusstherapiemaßnahmen zu beobachten als für Personen ohne solche Planung. Sie zeigen allerdings eine signifikant verlängerte rezidivfreie Phase nach Haftentlassung. Auch wenn über den Erfolg der Anschlussmaßnahme in dieser Studie keine Daten vorlagen, ist dies zumindest ein Hinweis für eine tatsächlich hohe Teilnahmerate an der vorgesehenen Anschlusstherapie.

Grundsätzlich stellt sich die Frage nach den inhaltlichen Kriterien einer Zugangsselektion für nachdrücklich geäußerte, d. h. in einem Einzelgespräch geprüfte, Teilnahmemotivation. Die abgelehnten Bewerber zeigen von der Tendenz her eine klar bessere Langzeit-

Tab. 5 Rezidivfreie Zeit nach Programmteilnahme/Bewerbung*(Signifikanztestung gegen Kontrollgruppe)

\begin{tabular}{|c|c|c|c|c|c|}
\hline & & $\begin{array}{l}\text { Rezidivlatenz } \\
\text { (Tage): } \\
\text { Gesamtkriminalität }\end{array}$ & $p$ & $\begin{array}{l}\text { Rezidivlatenz } \\
\text { (Tage): } \\
\text { Drogenkriminalität }\end{array}$ & $p$ \\
\hline \multirow[t]{3}{*}{ Programmteilnehmer insgesamt } & Mittelwert & 304 & 0,7 & 289 & 0,88 \\
\hline & Standardabw. & 412 & & 309 & \\
\hline & Median & 188 & & 186 & \\
\hline \multirow[t]{3}{*}{ Programmende regulär, ext. Therapieanschluss } & Mittelwert & $517^{* *}$ & 0,06 & $609^{* * *}$ & 0,17 \\
\hline & Standardabw. & 525 & & 643 & \\
\hline & Median & 338 & & 415 & \\
\hline \multirow[t]{3}{*}{ Programmende regulär, ohne ext. Therapieanschluss } & Mittelwert & 306 & 0,71 & 269 & 0,12 \\
\hline & Standardabw. & 392 & & 300 & \\
\hline & Median & 156 & & 145 & \\
\hline \multirow[t]{3}{*}{ Abbruch erste 100 Tage } & Mittelwert & 260 & 0,34 & 450 & 0,99 \\
\hline & Standardabw. & 272 & & 518 & \\
\hline & Median & 174 & & 281 & \\
\hline \multirow[t]{3}{*}{ Abbruch nach > 100 Tagen } & Mittelwert & 303 & 0,63 & 299 & 0,09 \\
\hline & Standardabw. & 425 & & 315 & \\
\hline & Median & 189 & & 217 & \\
\hline \multirow[t]{3}{*}{ Kontrollgruppe: abgelehnte Bewerber } & Mittelwert & 343 & & 450 & \\
\hline & Standardabw. & 505 & & 569 & \\
\hline & Median & 129 & & 263 & \\
\hline \multirow[t]{3}{*}{ insgesamt } & Mittelwert & 364 & & 440 & \\
\hline & Standardabw. & 458 & & 523 & \\
\hline & Median & 211 & & 252 & \\
\hline
\end{tabular}

*Startpunkt für Berechnung grundsätzlich Haftende $\quad{ }^{* *} \mathrm{p}=0,04$ 


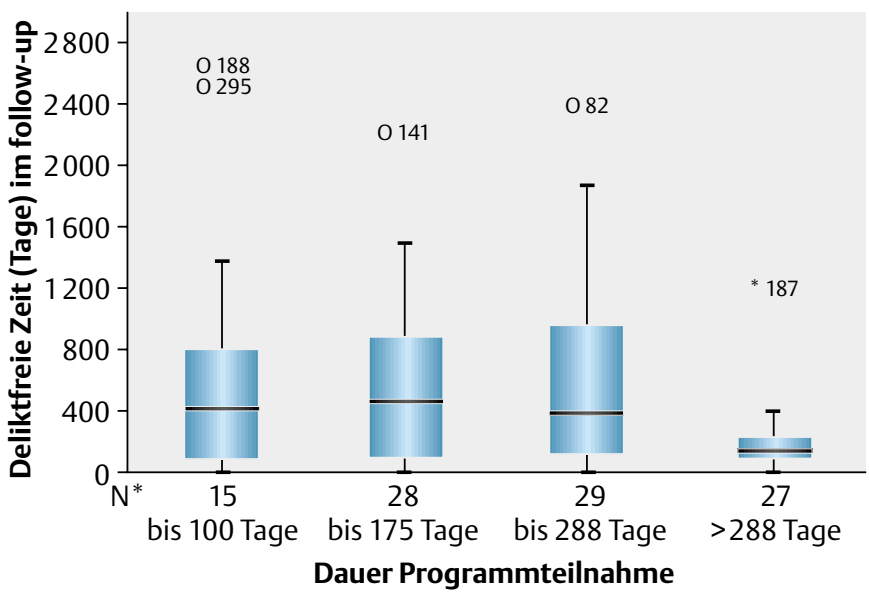

bewährung als Personen, die das Programm nach Zulassung vorzeitig beenden, und keine schlechtere als die Therapieteilnehmer insgesamt. Dies kann ein Argument dafür sein, das Angebot bedarfsdeckend auszuweiten. Personen mit frühzeitigem Abbruch sollten aufgrund der auffällig schlechten Kriminalprognose mit fast $100 \%$ Rezidiven verstärkt aufsuchende Beratungsangebote mit der Frage eines Wechsels der therapeutischen Ausrichtung, z.B. im Hinblick auf eine Substitutionsbehandlung im Vollzug mit Überleitung am Haftende, gemacht werden. Personen mit einem späten Abbruch dagegen unterscheiden sich z.B. hinsichtlich der Rückfalllatenz nicht wesentlich von Personen mit regulärem, aber spätem Programmabschluss (oberhalb 288 Tagen). Die Ergebnisse könnten insofern die Überlegung stützen, das Erprobungsprogramm bei Absenkung der Zugangsschwelle durchschnittlich zu verkürzen; bei parallel noch stärkerer Motivierung zur Wahrnehmung einer Anschlusstherapie. Mit gleichen Ressourcen würde so mehr abstinenzmotivierten Haftinsassen eine Chance eröffnet. Die hier vorgestellten Daten sind allerdings nicht geeignet, eine bessere Entscheidungsgrundlage über die Aufnahme in das Programm im individuellen Bewerberfall bereitzustellen, da in der Untersuchungsgruppe wesentliche soziostrukturelle Daten und die psychiatrische Komorbidität unbekannt waren.

Im Vergleich mit anderen publizierten Daten zeigt sich, dass Therapieprogramme mit einer ambitionierteren Interventionsphilosophie auf Basis individueller Therapiepläne gegenüber Kontrollgruppen vom Aspekt der Legalbewährung her besser abschneiden, wobei aber eine Vergleichbarkeit im engeren Sinne aufgrund unterschiedlicher Anwendung von Indikatoren und Follow-up-Zeiträume nicht gegeben ist.

$\mathrm{Zu}$ den wenigen bislang in Europa publizierten Studien zur Legalbewährung Drogenabhängiger nach abstinenzorientierten Programmen im Gefängnis bzw. nach Intervention überhaupt gehört die Longitudinalanalyse einer Therapeutischen Gemeinschaft der Jahre 1978 bis 1998 im Österaker Gefängnis in Schweden [19]. Das Konzept basiert auf Elementen kognitiver Verhaltenstherapie mit individuellen Therapieplänen. Auch hier sind tägliche Urintests üblich. Der Rehabilitationsprozess geht nach 8-10 Monaten bis zum erfolgreichen Abschluss gewöhnlich über in institutionell oder familiär gebundende Gemeinschaften für weitere 6-10 Monate. Ein Behandlungsansatz beinhaltet die kognitive Simulation und das Rollenspiel von Verführungsmomenten in Freiheit.
In einer ersten Auswertung der Jahre 1979 bis 1981 gelang es Insassen mit erfolgreichem Abschluss in 46\% der Fälle, in einer zweiten Studie bis 1996 in 66,6\%, Rückfälle zu vermeiden. Nach Dropouts betrugen die entsprechenden Raten 16\% bzw. 35,4\%. $45 \%$ aller Therapieteilnehmer wurden erneut straffällig im Vergleich zu 56\% einer Kontrollgruppe ohne spezialisiertes Programm. Die Autoren sehen die Reduktion subkulturellen kriminogenen Drucks auf die Teilnehmer als eine Ursache der Effektivität des Konzepts.

Die Evaluation von US-amerikanischen ITCs, die gegenüber dem übrigen Vollzug abgeschlossene Einheiten darstellen, zeigt seit den achtziger Jahren reduzierte Rückfall- und Reinhaftierungsraten (Cornestone-Programm: [12]; 1989: Stay'n out-Programm: $[13,14])$. Wexler [15] fasst jedoch zusammen, dass 9-12 Monate Mindestaufenthalt als Voraussetzung für eine Rückfallprophylaxe anzusehen seien.

Bei den neueren Projekten, die ITCs mit in der kommunalen Versorgung angesiedelten therapeutischen Gemeinschaften zu kombinieren (Kyle New Vision-Programm in Texas: [5, 6]; KEYCREST-Programm in Delaware: [7]; Amity-Programm in Kalifornien: [9]; New York: [10]), zeigt sich die Überlegenheit vor allem, je sequentieller die Programme aufgebaut und genutzt wurden $[16,17]$. 1-Jahres-Wiederinhaftierungraten betragen $25 \%$ [9] bei Komplettierung des Amity-Programms (unbehandelte Kontrollen 67\%). Günstig liegen auch Rückfallraten. Inciardi et al. [19] geben noch für ein 18-Monats-Follow-up eine Reinhaftierungsrate von nur 27\% (Kontrollgruppen: 55 bis 56\%) an. Die Programme unterscheiden sich in der für die ITC-Phase vorgesehenen Zeitdauer (zumeist 6-12 Monate), der Nutzung von verpflichtenden Übergangseinrichtungen (transitional therapeutic community, TTC) und der Dauer vorgesehener verpflichtender Teilnahme im kommunalen Nachsorgeprogramm. Grundsätzlich wird die Sinnhaftigkeit von peer-to-peer-orientierten Strategien in der Übergangsphase hervorgehoben [18]. Dies gilt v.a. bei Komorbidität mit einer dissozialen Persönlichkeitsstörung (im AmityProgramm bei $51 \%$ der Klienten). Das texanische Kyle-NewVision-Programm weist 6-Monats-Reinhaftierungsraten von nur $3 \%$ (Kontrollgruppe 15-16\%) nach. Dieses Programm wurde auch durch toxikologische Analysen von Haarproben im Follow-up evaluiert, die in der Gruppe derjenigen, die kombiniert ITC und TTC in Anspruch nahmen, in 35\% der Fälle Kokain zeigten (unbehandelte Kontrollgruppe 54\%, [19]). Während bislang Reinhaftierungsraten durch Nutzung von justizbehördlichen Haftregistern berechnet wurden, wird neuerdings ein höherer Differenziertheitsgrad der Auswertung durch selbstberichtete Delinquenz für künftige Follow-up-Evaluationen gefordert [18]. Grundsätzlich fällt auf, dass in den dargestellten Programmevaluationen Opiatabhängige zumeist nur Teilkollektive sind, für die eine eigene differenzierte Auswertung fehlt. Evaluationen auf Basis von randomisierten Studien mit Häftlingen gibt es bislang kaum; Ausnahmen sind die Effektmessung kognitiver Verstärkung als Interventionsstrategie in therapeutischen Gemeinschaften im Gefängnis [20] und pharmakologische Ansätze [21]. Insgesamt wird in der Literatur auf die Notwendigkeit verpflichtender Teilnahme an den kommunalen Nachsorgeprogrammen hingewiesen. 
Die Rückfallraten von Personen, die eine abstinenzorientierte Behandlung vorzeitig abbrechen, zeigen den hohen Bedarf für ein differenziertes Behandlungs- und Überleitungsangebot im Umfeld der Haftentlassung unter Einschluss der Substitution. Anderenfalls ist bei Haftende erneute Straffälligkeit aufgrund des Beschaffungsdrucks regelmäßige Folge. Dabei ist aber auch nicht zu verkennen, dass Drogenabhängige in Haft eine selektierte Gruppe darstellen, bei denen z.B. die primäre Zurückstellung der Haft zugunsten von Behandlungsmaßnahmen nicht möglich war, wegen einer besonders hohen Kriminalitätsbelastung, mangelnder Erreichbarkeit für Therapieorientierung oder vielfachem Scheitern von früheren Interventionsversuchen. Die in der vorliegenden Untersuchung besonders hohe Kriminalitätsvorbelastung im Vergleich zu Stichprobenuntersuchungen von Drogenabhängigen in ambulanter Abstinenztherapie in Freiheit (5-6\% Verurteilungen wegen neuer Delikte in einem einjährigen Follow-up [22] oder Substitution [23, 24]) ist so erklärbar und die Grundlage für eine eher niedrige Erwartung an die durchschnittliche Legalbewährung. Auf jeden Fall handelt es sich aber um einen grundsätzlich änderungs- und größtenteils auch therapiemotivierten Personenkreis. In dieser Studie fehlten Informationen über den konkreten Erfolg von Anschluss-Interventionen nach Entlassung. Auch die Therapiemotivierten unter der Kontrollgruppe haben möglicherweise zu einem größeren Anteil später von einer Entlassung nach $\S \S 35$ BtmG profitiert und zeigen auch aus diesem Grunde eine insgesamt durchschnittliche Legalbewährung. Es wäre ein Forschungsdesiderat, die Bedeutung von Anschlusstherapiemaßnahmen, gerade im Zusammenhang mit einer Vorphase der Abstinenzerprobung in Haft, auf ihre Effizienz sowie Outcome-Prädiktoren hin näher zu untersuchen. Auch diesbezüglich liegen in Deutschland bislang kaum wissenschaftliche Evaluationen vor.

\section{Literatur}

${ }^{1}$ Turnbull PJ, Mc Sweeney T. Drug treatment in prison and aftercare: A literature review and results of a survey of Euopean countries. Council of Europe: Drug misusing offenders in prison and after release. Strasbourg 2000; $41-59$

2 Stöver H, Merino PP. (Hrsg: EMCDDA). An overview study: Assistance to drug users in European Union prisons. Cranstoun Drug Services Publishing. London 2001

${ }^{3}$ Lapornik R, Zapotoczky HG. Value of drug screening in granting leave of absence to prisoners. Wien Klin Wochenschr; 110 (21): 755-758

${ }^{4}$ Jongerius JAHM, Koeter MWJ. Drang to verandering: Haalbaarheid en effecten van een drang-en dwangbenadering van verslaafden in detentie. Eerste fase. Amsterdam: AIAR 1997

${ }^{5}$ Knight K, Simpson DD, Chatham LR, Camacho LM. An assessment of prison-based drug treatment: texaś in-prison therapeutic community program. Journal of Offender Rehabilitation 1997; 24: 75-100
${ }^{6}$ Knight K, Hiller ML, Simpson D. Evaluating corrections-based treatment for the drug-abusing criminal offender. J Psychoactive Drugs 1999; 31: 299-304 (3)

${ }^{7}$ Martin SS, Butzin CA, Inciardi SA. Assessment of a multistage therapeutic community for drug-involved offenders. Journal of Psychoactive Drugs; $27:$ 109-116

${ }^{8}$ Wexler HK, De Leon G, Kressel D, Peters J. The Amity prison TC evaluation: reincarceration outcomes, Criminal Justice and Behavior. 1999; 26 (2): $147-167$

${ }^{9}$ Freudenberg N, Wilets I, Greene MB, Richie BE. Linking women in jail to community services: factors associated with rearrest and retention of drug-using women following release from jail. J Am Med Womens Assoc 1998; 53: 89-93 (2)

${ }^{10}$ Dolde G. Therapie in Untersuchungs- und Strafhaft. In: DHS, Gaßmann R (Hrsg). Suchtprobleme hinter Mauern. Drogen, Sucht und Therapie im Straf- und Maßregelvollzug. Lambertus 2002

${ }^{11}$ Untersuchungshaft- und Vollzugsanstalt Vierlande. Kurzinformation der Drogenfreien Stationen - Anstalt IX. Eigendruck. 1994

${ }^{12}$ Field G. The Cornerstone Program: a client outcome study. Federal Probation 1985; 49: 50-55

${ }^{13}$ Wexler HK, Williams R. The Staýn Out therapeutic community: prison treatment for substance abusers. Journal of psychoactive drugs 1986; 18: $221-230$

14 Wexler HK, Falkin GP, Lipton DS. Outcome evaluation of a prison therapeutic community for substance abuse treatment. Criminal Justice and Behavior 1990; 17: 71 - 92

${ }^{15}$ Wexler HK. The success of therapeutic communities for substance abusers in American prisons. Journal of Psychoactive Drugs 1995; 27 (1) : 57-66

${ }^{16}$ Nielsen AL, Scarpitti FR, Inciardi JA. Integrating the therapeutic community and work release for drug-involved offenders: the Crest program, Journal of Substance Abuse Treatment. 1996; 13: 349 - 358

17 Inciardi JA, Martin SS, Butzin CA, Hooper RM, Harrison LD. An effective model of prison-based treatment for drug-involved offenders. Journal of Drug Issues 1997; 27: $261-278$

${ }^{18}$ Hiller ML, Knight K, Simpson D. Prison-based substance abuse treatment, residential aftercare and recidivism. Addiction 1999; 94: $833-842(6)$

${ }^{19}$ Knight K, Hiller ML, Simpson DD, Broome KM. The validity of selfreported cocaine use in a criminal justice treatment sample. American Journal of Drug and Alcohol Abuse 1998; 24: 647-660

20 Newbern D, Dansereau DF, Pitre U. Positive effects on life skills motivation and self-efficacy: node-link maps in a modified therapeutic community. Am J Drug Alcohol Abuse 1999; 25 (3) : 407-423

${ }^{21}$ Cornish JW, Metzger D, Woody GE, Wilson D, McLellan AT, Vandergrift $B$, O'Brien CP. Naltrexone pharmacotherapy for opioid dependent federal probationers. J Subst Abuse Treatment 1997; 14 (6) : 529-534

${ }^{22}$ Raschke P, Verthein U, Kalke J. Ambulante Abstinenztherapie mit Drogenabhängigen. Freiburg i. Br: Lambertus 1996

${ }^{23}$ Raschke P. Legalbewährung von Substituierten und Substitution im Gefängnis. In: Krausz M, Raschke P (Hrsg). Drogen in der Metropole. Freiburg i. Br: Lambertus 1999

${ }^{24}$ Bathsteen M, Legge I. Intendierte und nicht intendierte Folgen des Hamburger Substitutionsprogramms. Z Kriminologie Strafrechtsreform 2001; 84: 1-9(1) 